MODELLING

\section{Stability from bistability}

It might be easier than we thought to make a neural integrator - at least according to work by Koulakov et al., published in Nature Neuroscience. They describe a new model of an integrator circuit that, unlike previous models, does not require precise tuning of synaptic strengths for stability.

Neural integrators can take shortterm inputs and produce lasting outputs, like cells in the frontal, temporal or parietal cortices that can show persistent spiking after a brief stimulus. This kind of sustained firing seems to be important in working memory, decision making and motor control.

Models of integrator circuits include tuned recurrent networks, in which constant firing rates result from reverberating neuronal activity. However, these circuits tend to be unstable unless the synaptic strengths are very finely tuned, and it is unclear whether such tuning is physiologically feasible. Koulakov et al. have overcome this problem in their models by using neural units that show bistability .

For a given input value, a bistable unit can have either of two stable output values. For example, imagine a neuron with a firing rate that increases as a function of input current. The threshold at which it starts to fire as current increases is higher than the level at which it stops firing when current decreases. Between these two values of input current, the neuron is bistable: it can either be

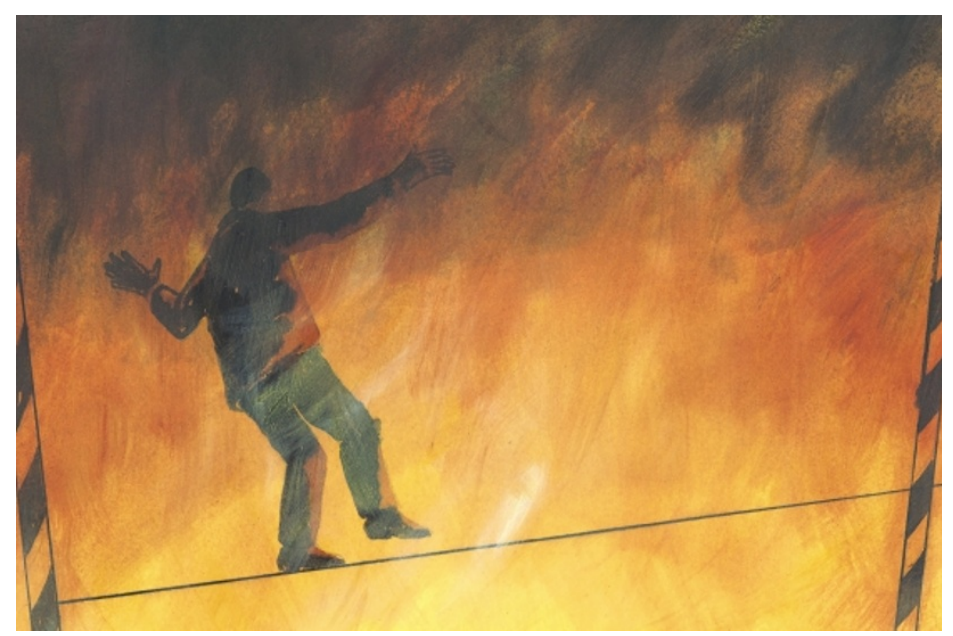

firing or not, depending on the recent history of inputs.

Koulakov et al. show that networks that are constructed from bistable units with overlapping 'bistable regions' of input can make much more robust integrators than did previous models that depended on synaptic tuning. They analyse two types of model: in one, bistability arises because each unit consists of an interconnected group of neurons that excite each other strongly; in the other, bistability in individual neurons results from the voltage-dependent properties of NMDA ( $N$-methyl-D-aspartate) receptors. Both models robustly integrate inputs, and are resistant to small changes in synaptic strength.

The idea that neural integrators could use bistability is still purely theoretical, but the authors list some predictions of the model that should be testable in experimental systems. For example, manipulations that reduce synaptic strength — such as treatment with low doses of receptor antagonists - should not affect integrator function. Testing such predictions, whether they prove to be true or false, will give us insight into integrators in the nervous system.

\section{Rachel Jones}

\section{(2) References and links} ORIGINAL RESEARCH PAPER Koulakov, A. A. et al. Model for a robust neural integrator. Nature Neurosci. 22 July 2002 (doi:10.1038/nn893) WEB SITES

Lisman's lab:

http://www bio brandeis.edu/lismanlab/

\section{IN BRIEF}

\section{SYNAPTIC PHYSIOLOGY}

Mossy fiber $\mathrm{Zn}^{2+}$ spillover modulates heterosynaptic $N$-methyl-D-aspartate receptor activity in hippocampal CA3 circuits.

Ueno, S. et al. J. Cell Biol. 158, 215-220 (2002)

Careful monitoring in hippocampal slices indicates that $\mathrm{Zn}^{2+}$ is released from mossy fibre terminals in an activity-dependent manner, and that it diffuses from there to the neighbouring stratum radiatum. This increase in $\mathrm{Zn}^{2+}$ seems to be responsible for the inhibition of NMDA-receptor-mediated synaptic responses that follows mossy fibre stimulation. The high concentrations of $\mathrm{Zn}^{2+}$ in mossy fibre terminals might modulate synaptic plasticity in nearby hippocampal networks. Although previous work had shown that $\mathrm{Zn}^{2+}$ could modulate signalling homosynaptically, it now appears that spillover of $\mathrm{Zn}^{2+}$ allows its effects to be more widespread.

\section{SYNAPTIC PLASTICITY}

Dendritic spikes as a mechanism for cooperative long-term potentiation.

Golding, N. L. et al. Nature 418, 326-331 (2002)

Golding et al. show that local, spatially restricted regenerative potentials, known as dendritic spikes, are produced by the activation of sodium and calcium channels following synaptic activation on distal dendrites of hippocampal CA1 pyramidal neurons. These spikes produce cooperative long-term potentiation by generating the postsynaptic depolarization and calcium entry that is needed to trigger potentiation, without requiring the backpropagation of action potentials.

\section{LIMBIC SYSTEM}

CREB activity in the nucleus accumbens shell controls gating of behavioral responses to emotional stimuli.

Barrot, M. et al. Proc. Natl Acad. Sci. USA 99, 11435-11440 (2002)

The authors show that both rewarding and aversive emotional stimuli activate CRE-mediated gene transcription in the nucleus accumbens shell. When the activity of CREB in this area was increased by gene transfer, reactions to rewarding, anxiogenic, aversive and painful stimuli were decreased, whereas a decrease in CREB caused increased responses. CREB seems to gate behavioural responses to emotional stimuli, regardless of their intrinsic value.

\section{CORTICAL DEVELOPMENT}

Conversion of cerebral cortex into basal ganglia in $E m \times 2^{-1-}$ Pax $6^{\text {Sey/Sey }}$ double-mutant mice.

Muzio, L. et al. Nature Neurosci. 5, 737-745 (2002)

The authors set out to discover whether two homeobox genes, Emx2 and Pax6, are required to subdivide the telencephalon into cerebral cortex and basal ganglia during development. In mice, inactivating either gene alone caused only mild defects, but when both genes were knocked out simultaneously, most of the cortical tissue was transformed into striatum. This result indicates that the products of just two genes stand between a cortical and a striatal fate. 